\title{
Flux Balance Analysis Based Model for the Identification of Potent Drug Target: A Novel Strategy
}

\author{
Bharath $\mathrm{BR}^{*}$ and Manjunatha $\mathrm{H}$ \\ Department of Biotechnology and Bioinformatics, Jnanasahyadri, Kuvempu University, Shankaraghatta,
} Karnataka, India

\begin{tabular}{|c|c|}
\hline Abstract & Article Information \\
\hline \multirow{3}{*}{$\begin{array}{l}\text { The work aims at developing a strategy for the identification of potent drug } \\
\text { target by analyzing signaling pathways. The strategy developed is designed } \\
\text { and tested in concern with Toll Like Receptor-4 (TLR4) signalling pathway. } \\
\text { TLR4 pathway is a major pathway which get trigger against Lipo- } \\
\text { polysaccheride (LPS) stimulus. The work describes a strategy to identify } \\
\text { potent drug targets by understanding the flow of information in signaling } \\
\text { pathways by representing them in the form of stoichiometric matrices and } \\
\text { analyzing mathematically by adopting relatively successful approaches, } \\
\text { including flux balance analysis (FBA), Single Value Decomposition (SVD). The } \\
\text { strategy successfully proposes TNF- } \alpha \text { Receptor Associated Factor-6 (TRAF6) } \\
\text { as a potent drug target for LPS neutralization. }\end{array}$} & $\begin{array}{l}\text { Article History: } \\
\text { Received : :05-02-2013 } \\
\text { Revised : 27-03-2013 } \\
\text { Accepted : 28-03-2013 } \\
\end{array}$ \\
\hline & $\begin{array}{l}\text { Keywords: } \\
\text { LPS } \\
\text { TLR4 } \\
\text { FBA } \\
\text { TRAF6 } \\
\end{array}$ \\
\hline & $\begin{array}{l}{ }^{*} \text { Corresponding Author: } \\
\text { Bharath BR } \\
\text { E-mail: } \\
\text { bharathbasavapattan@gmail.com }\end{array}$ \\
\hline
\end{tabular}

\section{INTRODUCTION}

Fundamental cell processes would be driven by intercellular and intracellular communication via direct contact or soluble factors like hormones or cytokines. Environmental signals received by membrane molecules called receptors, which induce cascade of biochemical reactions in cell cytoplasm and nucleus (Alberts and Johnson, 2002). In effect, usually the expression level of target genes has altered, that corresponds to cell response to the input stimuli. The intracellular reactions occur between biomolecules, through fixed, in a timescale of single signal processing, set of reaction channels. Cell response has determined by transient dynamics of such interactions network by pathway engineering (Rowinsky, 2003).

Pathway engineering is to optimize pathway networks to reveal and understand the signal flow in networks. To reach this goal, quantification of the pathway network is necessary, to quantify there is a need to measure the fluxes in the pathway network. Many high throughput experiments are in use for the analysis of cellular mechanisms. Main drawback of the high throughput biological experiments is its results data bias and detailed inquiring into specific interactions or their kinetic data (Cho and Labow, 2006). Scientific computing and mathematical modeling of such molecular, signal processing pathways is very beneficial in acquiring system level understanding about dynamical mechanisms of cell (Butcher and Berg, 2004).

In that connection several modeling approaches have been used over recent years to analyze complex biological systems such as signaling pathways, ranging from traditional mathematical methods based on differential equations to computational methods based on stochastic simulation and model checking (Regev et al., 2001).

Although there are a variety of metabolic modeling approaches, all of them share a fundamental requirement: a stoichiometric matrix based on a reconstructed metabolic network. Each column of the stoichiometric matrix 
Bharath et al.,

corresponds to a chemical or transport reaction, with non-zero values that identify the metabolites which participate in the reaction as well as the stoichiometric coefficients that correspond to each metabolite. The matrix also contains directionality: substrate and product metabolites in the matrix have negative and positive coefficients, respectively. By considering the matrix rows instead of the columns, the stoichiometric matrix can also be thought as the list of reactions in which a given metabolite participates. This interpretation is useful while defining mass balances for each metabolite in the network.

Metabolic Flux Balance Analysis (FBA) is one such a method for analyzing the flow of metabolites through a metabolic network (Covert et al., 2001). It is a mathematical method for analyzing metabolism. It is a direct application of linear programming to biological systems that uses the stoichiometric coefficients for each reaction in the system as the set of constraints for the optimization. Additionally, this method requires the assumption of biological steady state (Covert and Palsson, 2003). Imposing this restriction allows the assumption that at any given time the concentration of a given compound in the metabolic network is constant (Covert et al., 2001).

Except in some modified forms, FBA does not account for regulatory effects such as activation of enzymes by protein kinases or regulation of gene expression. In that regard, the current study proposes a model to predict potential drug target for LPS neutralization by considering regulatory effects in signaling as reactions and the study focuses on the concept of signal crosstalk or cancelation observed in signaling cascades to identify the potent drug target by adopting SVD method.

\section{MATERIALS AND METHODS}

\section{Pathway Retrieval and Analysis}

For the representation of a flux there is a need to visualize and understand the pathway, this has achieved by the retrieval of TLR4 pathway from the pathway database PANTHER (http://www.pantherdb.org/pathway/) in *.SBML (System Biology Markup Language) format. Cell Designer V3.0 has used for Analysis and reconstruction of pathway (Bornstein and Keating, 2008).

\section{Pathway Network Construction}

Signals in a pathway are represented with the help of signaling network. The most important
Sci. technol. arts Res. J., Jan-Mar 2013, 2(1): 50-55

assumption made for the FBA is that the model will be in a steady state. The steady state of signaling networks can be described in terms of mass balance equations. These equations describe the change of signal flux over time, based on the fluxes through the signaling network. The internal fluxes are represented by " $V$ " and the external fluxes are represented by " $b$ ". The signals in TLR pathway were identified and pathway network is constructed, the mass balance for is represented with the help of stoichiometric matrix $\mathrm{S}$ with the flux vector $\mathrm{V}$.

\section{Stoichiometric Matrix Construction}

A stoichiometric matrix $S$ is an $m^{*} n$, where $m$ corresponds to the number of genes and $n$ is the number of chemical reactions or fluxes taking place within the metabolic network. The vector $v$ corresponds to the quantity of fluxes within the signaling network. It also describes the rate at which the gene is chemically signaling to the gene at downstream. The fluxes, which enter the gene instances, were considered positive and the fluxes that leave the genes are considered negative. In the study, for simplification, the fluxes are considered to be either "1" (Signal input) or "-1" (Signal output). Optimization of the stoichiometric matrix was done to optimize the metabolic network by which a potent drug target for LPS neutralization can be identified. Singular Value Decomposition (SVD) has conducted to identify the dependent Extreme paths in the network usin MATLAB.

\section{SVD Using MATLAB}

Mass balance equations are represented as linear equations. The null spaces need to be obtained which is a set of operands which solves

$$
\text { S.v= } 0 \text { (Orth et al., 2010) }
$$

This can be determined by employing SVD method. MATLAB is a programming environment for algorithm development, data analysis, visualization, and numerical computation. The following coding is used to optimize the stoichiometric matrix:

Algorithm for the execution of SVD for the derived matrix:

1. Input matrix values, Matrix_name $=X$.

2. Calculate the matrix by calling $\operatorname{rank}()$ and assign the value to the variable $R$.

3. Derive SVD by calling svd() and assign values to the matrices $U, S$ and $V$ respectively.

4. Derive BETA, the final vector matrix by calling null (Matrix_name,'r').

5. The graphs are plotted by using code Plot(Matrix_name). 
Bharath et al.,

\section{RESULTS}

The TLR pathway is retrieved from PANTHER pathway database and critically analyzed by
Sci. technol. arts Res. J., Jan-Mar 2013, 2(1): 50-55

employing Cell Designer V3.0 as shown in Figure 1.

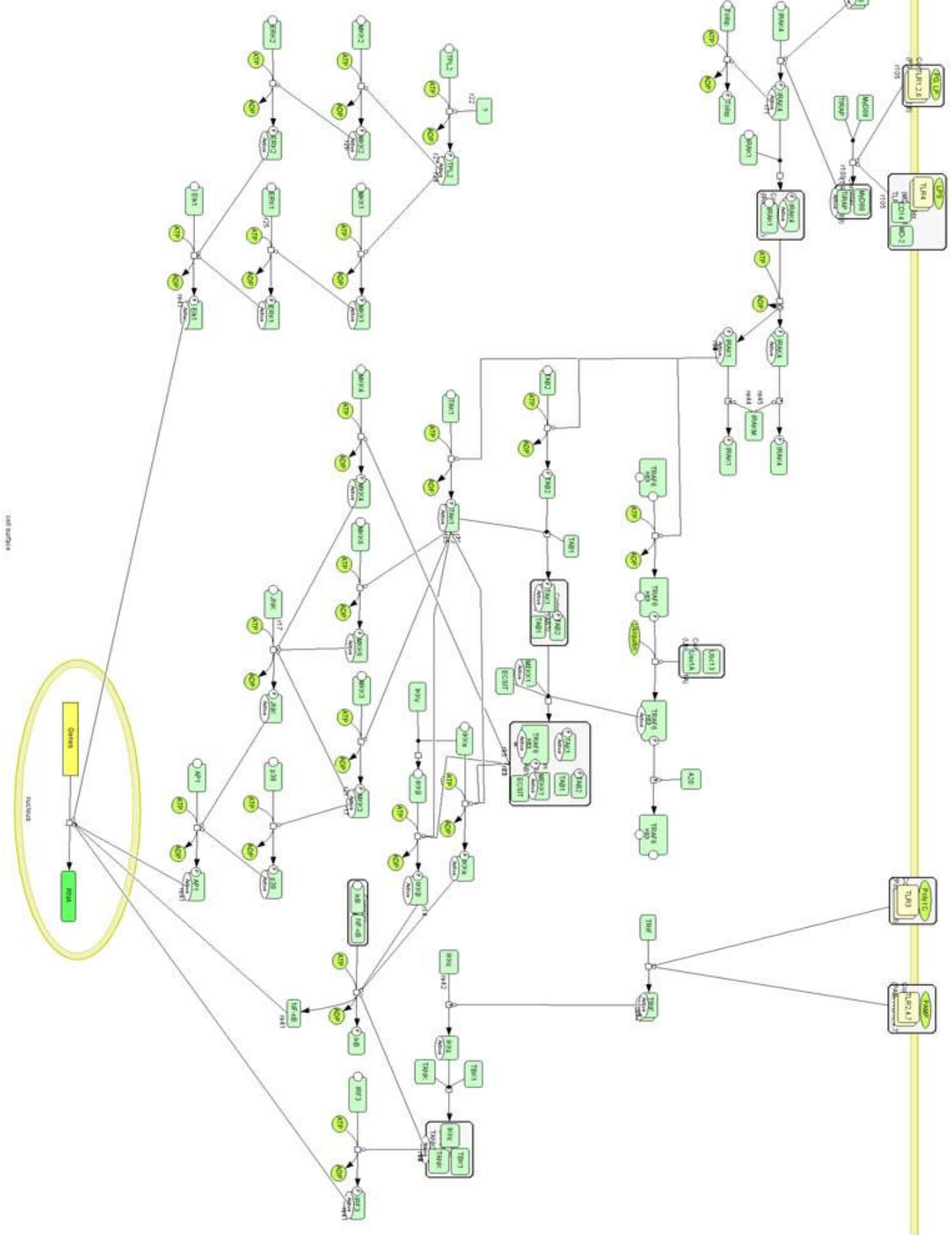

Figure 1: TLR4 signaling pathway visualized using Cell Designer. 
Bharath et al.,

The stoichiometric matrix $\mathrm{S} 1$ is derived for TLR4 pathway shown in Figure 2A accordingly reconstructed pathway model is shown in Figure $2 \mathrm{~B}$ by considering flux incoming as 1 , no reaction

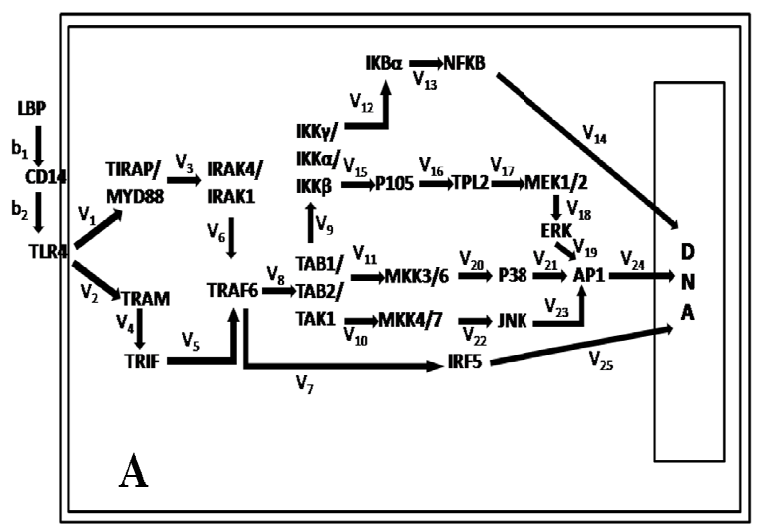

Sci. technol. arts Res. J., Jan-Mar 2013, 2(1): 50-55 as 0 and outgoing flux as -1 . The stoichiometric matrix-1 (S1) holds the information of TLR4 pathway mathematically.

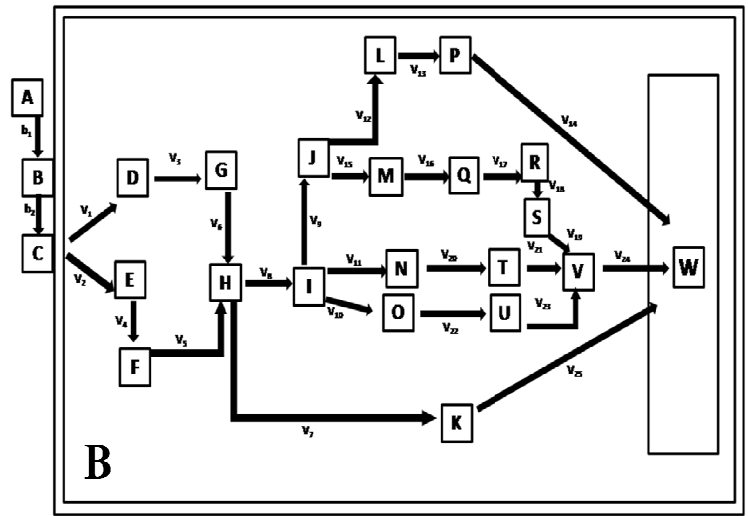

k, (B) Reconstructed pathway model.
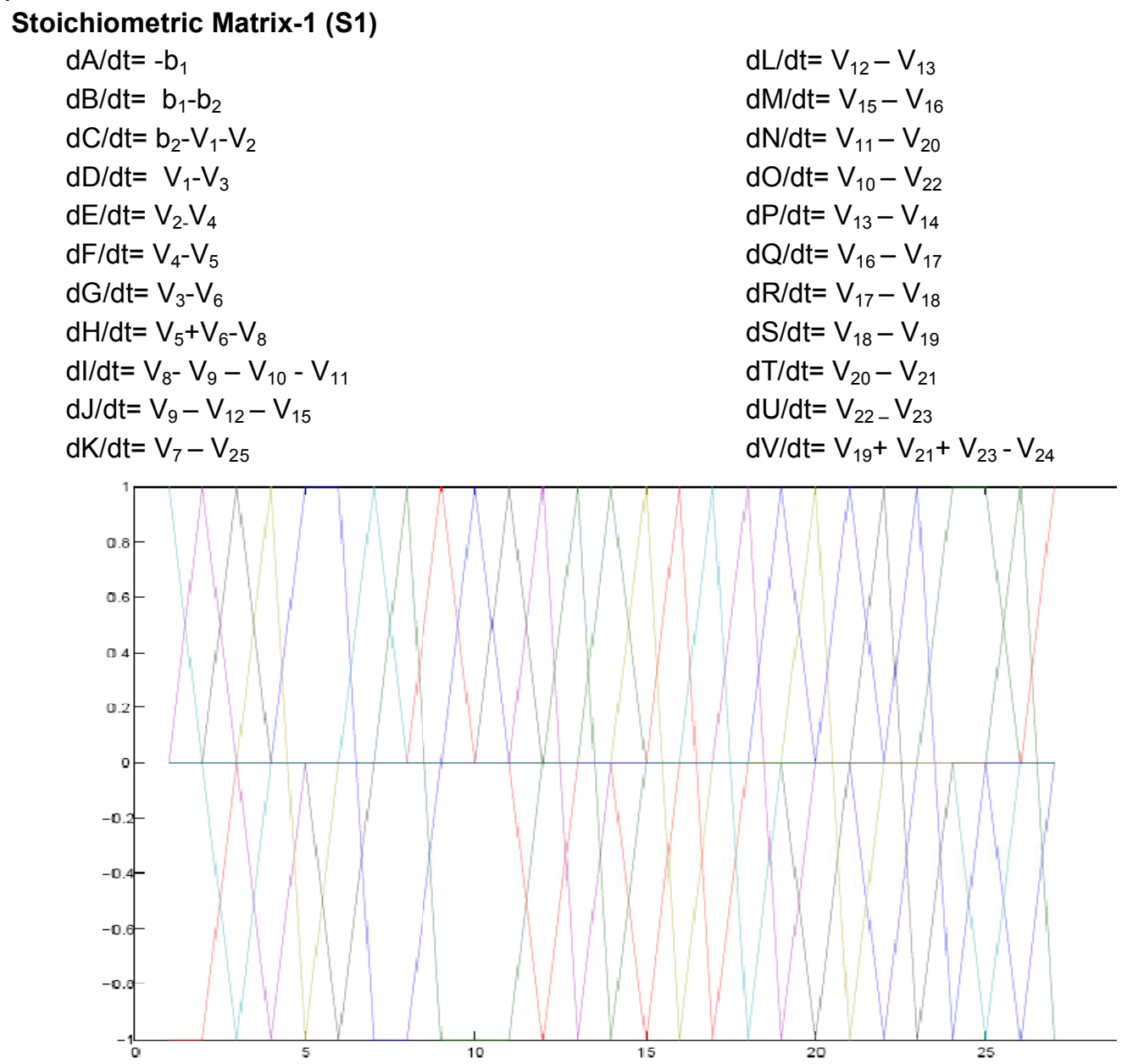

Figure 3: Representation of $S 1$ in the form of graph, here 27 vectors (2 extrinsic and 25 intrinsic) are taken horizontally. Each vector touches $-1,0$ and 1 for outgoing, no reaction and incoming reactions respectively. 


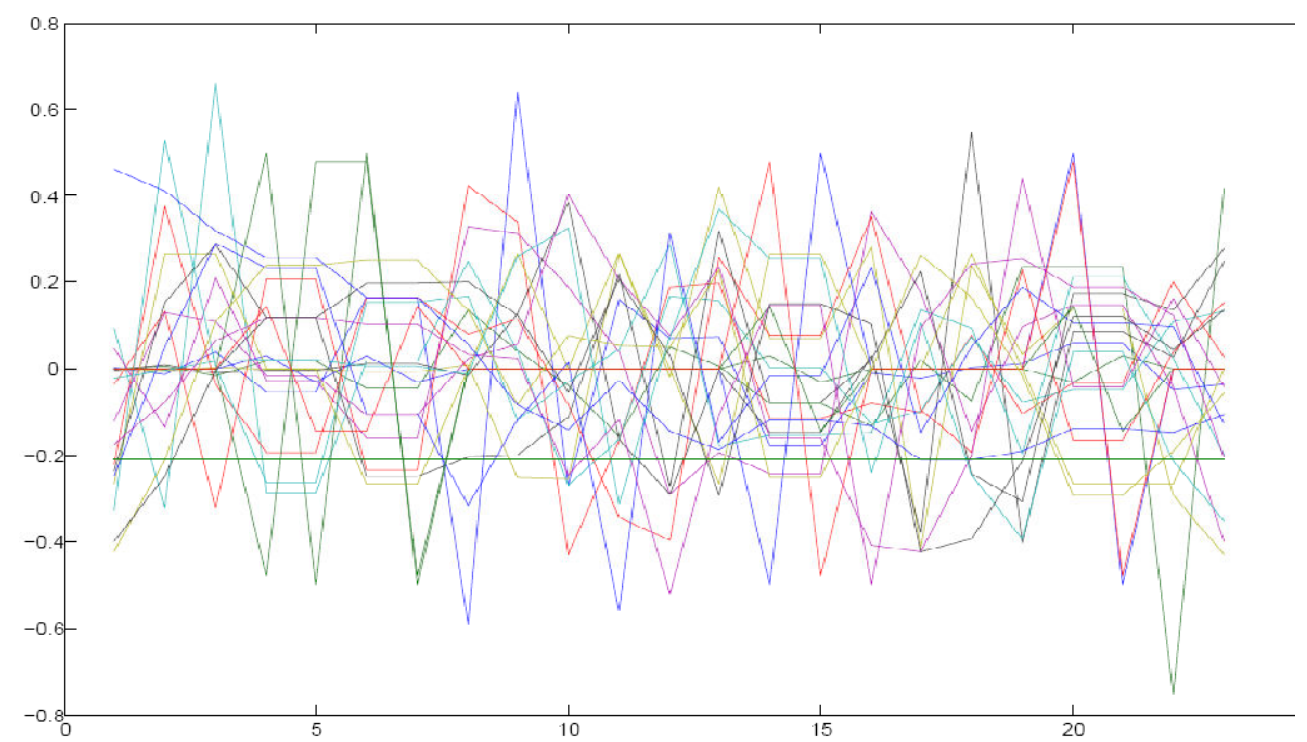

Figure 4: Representation of matrix $V$ after SVD in the form of graph, here 27 vectors $(2$ extrinsic and 25 intrinsic) are taken horizontally. Each vector shows their flux level in pathway network, Node $\mathrm{V}$ and Node $\mathrm{H}$ shares least flux -0.59 (blue line)and -0.75 (green line) respectively.

\section{DISCUSSION}

Drug currently used in the market to treat many diseases have limited therapeutic value due to the failure in identifying a best therapeutic drug targets. The target identification needs the proper understanding of the pathways involved in disease. Rapidly increasing number of revealed biological pathways calls for the development and use of simulation methods and tools that would facilitate their analysis \& virtual experimentation. Metabolic network reconstruction and simulation allows for an in depth insight into comprehending the molecular mechanisms of a particular organism, especially correlating the genome with molecular physiology.

In that concern the present work contributes as a novel interdisciplinary approach for the representation of engineered models in a consistent format and further simulating them in silico, for the drug target identification, standardization and model formalism. Here the knowledge on signaling pathways involved in TLR4 pathway is downloaded from the PANTHER pathway database and critically analyzed by employing the pathway visualization tool Cell Designer V3.0 as shown in Figure 1. A closer assessment of pathways revealed that signaling pathways interact with each other, forming a network. Complexity in pathway network is reduced and common genes were identified in order to reveal the role of all the potential paths in signaling pathway for crosstalk. Few genes, which can contribute for the signaling, were manually curated and added using the Cell Designer V3.0.
The critical analysis of pathways encouraged us to design the pathway networks as shown in Figure 2A. The initial biological pathway models are reconstructed as pathway models to reduce the complexity. Pathway models are constructed by assuming every nodes in signal as English alphabets, internal reactions as $V_{i}$ and external reactions as $b_{i}$ as given in the Figure 2B. These pathway models facilitated the construction of stoichimetric matrix of TLR4 pathway with 23 rows and 27 columns having rank of matrix 22 .

The stoichimetric matrix is subjected for the SVD analysis for the identification of extreme pathways. SVD method derived the extreme pathways for $\mathrm{S} 1$ with 23 rows and 23 columns. In the derived matrix node $\mathrm{H}$ and node $\mathrm{V}$ shared least flux value of -0.59 and -0.75 respectively, this very clearly indicates that node $\mathrm{H}$ and node $\mathrm{V}$ consisting of more outgoing reactions in all possible extreme paths when compare with other nodes in the same pathway. The cross verification for nodes $\mathrm{H}$ and $\mathrm{V}$ through the original pathway found matched with TRAF6 and AP1, it clearly reveals TRAF6 and AP1 can act as probable drug targets. Since AP1 is a nuclear factor which has the accessibility into nuclear membrane it found risky to consider it as the drug target, thus TRAF6 can be strongly proposed as potent drug target for LPS neutralization. The identified drug target is validated by manual text mining method; the earlier investigators also reported TRAF6 as a potent drug target for LPS neutralization. 
Bharath et al.,

\section{CONCLUSIONS}

The method proposed in the study found reliable for all the pathways to identify a potent drug target. Degree of error in the method depends on sensitivity of pathway construction, the error in pathway construction reflects on method sensitivity.

\section{REFERENCES}

Alberts, B., Johnson, A. (2002). Molecular Biology of the Cell. Garland, $4^{\text {th }}($ Edn.).

Bornstein, B.J., Keating, S.M. (2008) LibSBML: An API Library for SBML. Bioinformatics, 880-881.

Butcher, E.C., Berg, E.L. (2004). Systems biology in drug discovery. Nature Biotechnology, 22, 12531259.

Cho, C.R., Labow, M. (2006). The application of systems biology to drug discovery. Current Opinion in Chemical Biology, 10, 294-302.
Sci. technol. arts Res. J., Jan-Mar 2013, 2(1): 50-55

Covert, M.W., Palsson, B.O. (2003). Constraints-based models: regulation of gene expression reduces the steady-state solution space. Journal of Theoretical Biology, 221, 309-325.

Covert, M.W., Schilling, C. H., Palsson, B. (2001). Regulation of gene expression in flux balance models of metabolism. Journal of Theoretical Biology, 213, 73-88.

Orth, J.D., Thiele, I., Palsson. B.O. (2010). What is flux balance analysis. Nature Biotechnology, 28, 245248.

Regev, A., Silverman, W., Shapiro, E. (2001). Representation and simulation of biochemical processes using the -calculus process algebra. In Proceedings Pacific Symposium on Biocomputing. 459-470.

Rowinsky, K. E. (2003). Signal Events: Cell Signal Transduction and Its Inhibition in Cancer, The Oncology, 8, 5-17. 